\title{
Advanced Alignment of the ATLAS Tracking System
}

\author{
Sebastian Pedraza-Lopez on behalf of the ATLAS Collaboration. IFIC (CSIC-UV)
}

\begin{abstract}
In order to reconstruct trajectories of charged particles, ATLAS is equipped with a tracking system built using different technologies embedded in a $2 \mathrm{~T}$ solenoidal magnetic field. ATLAS physics goals require high resolution and unbiased measurement of all charged particle kinematic parameters. These critically depend on the systematic effects related to the alignment of the tracking system. In order to eliminate malicious systematic deformations, various advanced tools and techniques have been put in place. These include information from known mass resonances, energy of electrons and positrons measured by the electromagnetic calorimeters, etc. Despite being stable under normal running conditions, ATLAS tracking system responses to sudden environmental changes (temperature, magnetic field) by small collective deformations. These have to be identified and corrected in order to assure uniform, highest quality tracking performance throughout the whole data taking. We will present an outline of the track based alignment approaches and special attention will be paid to techniques allowing to identify and eliminate tracking systematics.
\end{abstract}

\section{INTRODUCTION}

$\mathbf{T}$ HE Large Hadron Collider (LHC) produces protonproton collisions at unprecedent centre-of-mass energies. The ATLAS detector is recording the products of these collisions in order to make precise measurements of Standard Model processes as well as to search for new physics phenomena beyond the Standard Model [1].

In order to reconstruct the trajectories of charged particles, ATLAS relies on an Inner Detector (ID) tracking system which provides an efficient, robust and precise tracking. The track reconstruction accuracy is intrinsically limited by the finite resolution of the sensitive devices, therefore, any detector misalignment adds to the uncertainty on the hit position and consequently on the reconstructed track parameters. It is known that certain correlated geometry distortions lead to systematic biases on the reconstructed track parameters, these are particulary harmful as they can lead to distortions from some of physics measurements.

The ID has been aligned using a track-based technique, however, any generic track-based alignment is confronted with some modes to wich fitted tracks have very low or no sensitivity, these so called weak modes of alignment are detector deformations that preserve a helical trajectory of the tracks and hence do not affect the $\chi^{2}$ of the track fit. Weak modes are the principal source of systematics effects related to the alignment procedure.

\section{The ATLAS InNer Detector}

The Inner Detector tracking system was designed to provide efficient and robust track reconstruction for charged particles.
It consist of three subdetectors: Pixels, SCT and TRT. The Pixel and SCT are silicon based detectors but using two distinct technologies, pixels and microstrips respectively. The TRT is a drift chamber composed of straws filled with gas.

The entire tracking system is embedded within a superconducting solenoid coil which produces a $2 \mathrm{~T}$ axial magnetic field.

\section{A. The Pixel detector}

The Pixel detector is the innermost subdetector in ATLAS. Its first layer is located just $5 \mathrm{~cm}$ from the nominal beam axis. The detector consist of 1744 silicon pixel modules arranged in three concentric barrel layers and two end-caps of three disks each. The active area of each module is $16.4 \mathrm{~mm} \times 60.8$ $\mathrm{mm}$, while the size of each pixel is $50 \mu \mathrm{m} \times 400 \mu \mathrm{m}$. The resolution in the $r \phi$ (module local $x$ ) direction is $\sim 10 \mu \mathrm{m}^{1}$, whilst $\sim 115 \mu \mathrm{m}$ is achieved in the module local $y$ direction.

\section{B. The silicon micro-strip tracker (SCT)}

The SCT modules are built from two pairs of single-sided silicon micro-strip sensors resulting in a $12 \mathrm{~cm}$ long module. The pairs of sensors are glued back-to-back with a relative stereo angle of $40 \mathrm{mrad}$ which allows for the measurement of both coordinates. A total of 4088 SCT modules are used to form four concentric barrel layers (2112 modules) and $2 \times 9$ end-cap disks (1976 modules). All barrel modules are identical, whilst there are 4 types of end-cap modules. The barrel modules have a rectangular shape with a constant strip pitch of $80 \mu \mathrm{m}$. The end-cap silicon sensors have a wedge shape with a constant inter-strip angle with an average strip pitch of $80 \mu \mathrm{m}$. The resolution of the SCT modules in $r \phi$ (module local $x$ ) direction is $\sim 17 \mu \mathrm{m}$, whilst $\sim 580 \mu \mathrm{m}$ is achieved in the module local $y$ direction.

\section{TRT}

The TRT is the outermost and the largest of the ID subdetectors. It consists of 298,304 proportional drift tubes (straws). Each straw has a diameter of $4 \mathrm{~mm}$ and has a sense wire in its center. The straw tubes are grouped together in 176 modules. The barrel part contains 96 modules, arranged in 3 barrel layers with 32 modules each, and 72 layers of straws. Each of the TRT end-caps consist of 20 modules with a total 160 planes of straws. The TRT straw tubes have a singlehit resolution of $\sim 130 \mu \mathrm{m}$ from the drift-time measurement.

\footnotetext{
${ }^{1}$ Studies in neural network clustering reconstruction improve the pixe resolution in the $r \phi$ direction up to $\sim 5 \mu \mathrm{m}[2]$
} 
The TRT aslso provides electron identification by detection of transition radiation photons emitted in the polypropylene fibers (barrel) or foils (end-caps) interleaved between the straws.

TABLE I

SUMMARY OF THE MAIN CHARACTERISTICS OF THE THREE ATLAS ID SUBDETECTORS [1].

\begin{tabular}{ccccc}
\hline Subdetector & $\begin{array}{c}\text { Radius } \\
{[\mathbf{c m}]}\end{array}$ & Element size & $\begin{array}{c}\text { Resolution } \\
{[\mu \mathrm{m}]}\end{array}$ & $\begin{array}{c}\text { Hits/track } \\
\text { barrel }\end{array}$ \\
\hline Pixel & $5-12$ & $50 \mu \mathrm{m} \times 400 \mu \mathrm{m}$ & $10 \times 115$ & 3 \\
SCT & $30-52$ & $80 \mu \mathrm{m}$ & 17 & 8 \\
TRT & $56-107$ & $4 \mathrm{~mm}$ & 130 & 30 \\
\hline \hline
\end{tabular}

\section{The Inner Detector alignment}

In order to achieve the ATLAS physics goals the degradation of the track paramenters due to misalignments should be less than $20 \%$ with respect to the intrinsic tracker resolution. The detector alignment should obtain the corrections of the modules positions in order to describe accurately the real detector. The strategy of the ID alignment has three parts. The first one uses the initial knowledge of the module positions based on surveys done during the assembly. The second one is composed by track-based offline algorithms and the last one is the validation with alignment and physics observables.

The track-based offline alignment algorithms are based on the residual $\chi^{2}$ minimization:

$$
\chi^{2}=\sum_{t r k}\left[\mathbf{r}^{T}(\tau, \mathbf{a}) V^{-1} \mathbf{r}(\tau, \mathbf{a})\right]
$$

where the $\mathbf{r}$ is the vector of track-hit residuals and $\mathrm{V}$ is the covariance matrix of the detector measurements. The residuals are a function of the track parameters $(\tau)$ and the alignment parameters (a). Then one has to minimize the $\chi^{2}$ with respect of the alignment parameters, wich are 6 degrees of freedom (DoF) per module:

$$
\frac{d \chi^{2}}{d \mathbf{a}}=0 \longrightarrow \sum\left[\mathbf{r}^{T} V^{-1}\left(\frac{\partial \mathbf{r}}{\partial \boldsymbol{\tau}} \frac{d \boldsymbol{\tau}}{d \mathbf{a}}+\frac{\partial \mathbf{r}}{\partial \mathbf{a}}\right)\right]=0
$$

with:

$$
\boldsymbol{\tau}=\left(d_{0}, z_{0}, \phi_{0}, q / p\right)
$$

where $d_{0}$ and $z_{0}$ are the transverse and longitudinal impact parameters respectively, measured with respect the ATLAS nominal interaction point. The angle $\phi_{0}$ is the azimuthal angle of the track at the perigee (the distance of closest approach to the nominal interaction point), and $\theta$ is its polar angle. The ratio $q / p$ is the inverse of the particle momentum multiplied by its charge.

The full ID alignment is a challenging problem due to the large number of degrees of freedom (see table II), so the alignment is performed in three different Levels of granularity: Level 1 dealing with entire subsystems or their central and endcaps parts, Level 2 concerned with barrel layers and discs for silicon detectors and barrel modules or end-cap wheels for the TRT, and finaly L3 dealing with individual silicon modules and TRT straws [3].
TABLE II

MAIN CHARACTERISTICS OF THE DIFFERENTS ALIGNMENT LEVELS.

\begin{tabular}{c|ccc|cc|c}
\hline \hline Levels & \multicolumn{3}{|c|}{ Structures } & \multicolumn{2}{c|}{ DoFs } & $\begin{array}{c}\text { Typical } \\
\text { misalignment } \\
{[\mu \mathrm{m}]}\end{array}$ \\
\hline Level 1 & PIX & SCT & TRT & Silicon & TRT & 1000 \\
Level 2 & 9 & 22 & 3 & 24 & 17 & 100 \\
Level 3 & 1744 & 4088 & 350848 & 34992 & 701696 & 10 \\
\hline \hline
\end{tabular}

\section{E. Time dependent alignment}

The Inner Detector is aligned at Level 1 in a run by run basis. We can observe that the detector is sensitive to changes in working conditions as it is shown in Fig. (1). Large movements of the detector are measured after hardware incidents, but between these periods the detector is generally very stable.

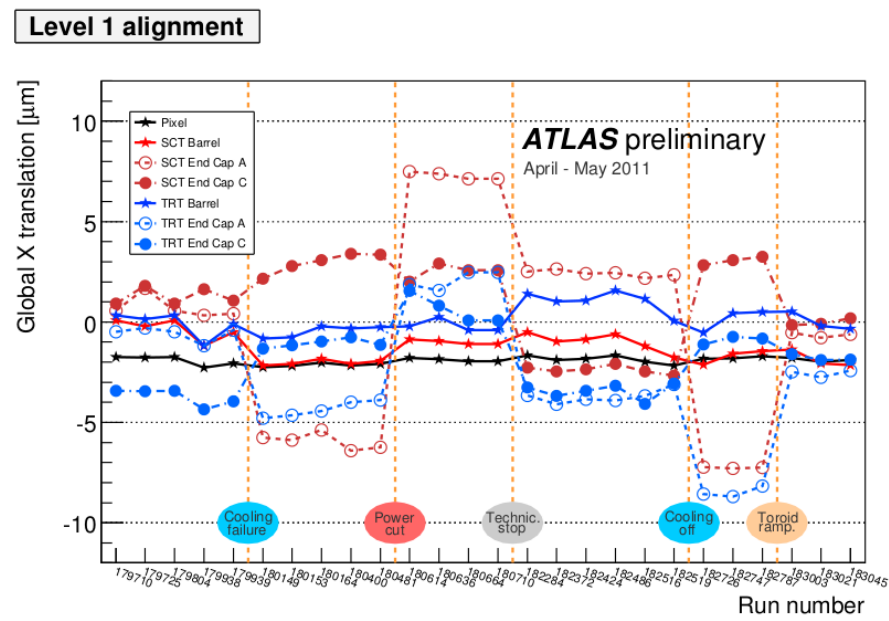

Fig. 1. Level 1 translations corrections in the global $\mathrm{x}$ direction versus run number [3]

\section{SYSTEMATICS DEFORMATIONS}

Any correctly constructed alignment algorithm is capable of producing a description of the detector geometry which provides an efficient track fit, but it is far more difficult to guarantee that the track parameter reconstruction is free from systematic biases [4] .

It is posible to find geometry descriptions which satisfy the assumed track model but lead to biased physics measurements. These are commonly called the weak modes of alignment as they correspond to near-singular modes of the solution to the alignment problem. The weak modes can be also defined as the geometry deformations to which track-hit residuals or the $\chi^{2}$ of the track fit remain invariant. They can arise from deformations of the actual detector or as artefact of the alignment procedure itself.

The track parameters for which it is easiest to introduce significant distortions and which consequently have most significant influence on physics measurements are the measured particle momentum and the track impact parameter. The weak mode deformations cannot be identified just by studing the quality of individual track fits. In order to identify these 
deformations, one needs either additional information or to rely on measurements which are universal and dependent on the reconstructed track parameters.

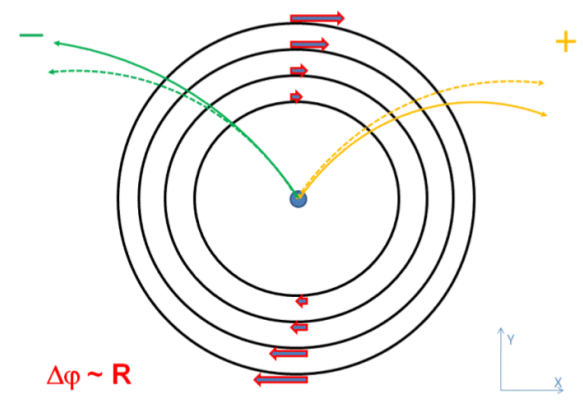

Fig. 2. Example of basic and simplest distortion affecting the measured particle momentum in a charge-antisymmetric way, the curl. The detector deformation as well as the impact on the reconstructed particle momenta are shown schematically. The true particle trajectories are shown as dashed lines, and the reconstructed trajectories as continuous lines. In general, the systematic deformations of the detector will not be as simple as this example. Due to limited correlation between the modules during the alignment procedure, higher order deformations are foreseen and would result in localized distortions to the track parameters [4].

In the next sections it will be shown the methods we use to find and correct any posible systematic deformation, like the measuring of both momentum and impact parameters bias, as well as the results.

\section{A. Measurement of detector distortions using the electron $E / p$ method}

By using the electromagnetic calorimeter as a reference system, it is posible to probe the systematics present in the ID by using the ratio of measured energy deposited in the calorimeter $(E)$ and of momentum as measured by the ID $(p)$ of electrons and positrons. Under the assumption that the calorimeter response is independent of the charge of the incoming particle, charge-dependent momentum biases introduced by the alignment procedure in the ID are expected to be seen as differences in $E / p$ ratio for electrons and positrons.

A sagitta deformation in the Inner Detector would bias the reconstructed momentum and the measured $\langle E / p\rangle$ should be modified as:

$$
<E / p>^{ \pm} \longrightarrow<E / p>^{ \pm}+q<E_{T}>\delta_{\text {sagitta }}
$$

If it is assumed that $<E / p>_{\text {true }}^{+}=<E / p>_{\text {true }}^{-}$, then it is possible to extract the momentum biases present in the detector. This is a valid assumption if the kinematic properties of the selected electrons and positrons are similar, because of that, electrons from $W$ and $Z$ boson decays have been used to obtain a high purity sample of electrons candidates. Therefore the momentum bias in terms of $\langle E / p>$ is:

$$
\delta_{\text {sagitta }}=\frac{<E / p>_{r e c}^{+}-<E / p>_{r e c}^{-}}{2<E_{T}>}
$$

Then we correct the reconstructed track parameter $q / p$ using this measured momentum bias:

$$
q / p_{\text {Corrected }}=q / p_{\text {reconstructed }}\left(1-q p_{T} \delta_{\text {sagitta }}\right)
$$

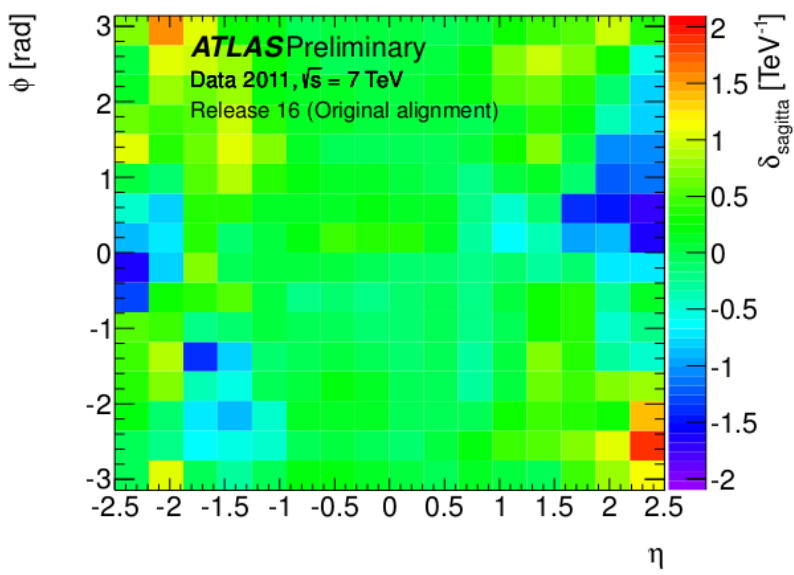

(a) Before alignment

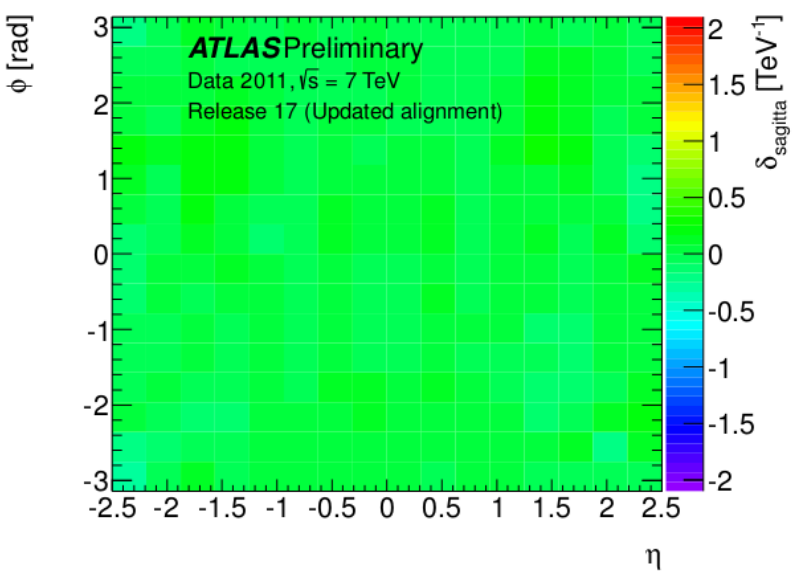

(b) After aligment

Fig. 3. Momentum bias measured before and after momentum constrained alignment. Using $E / p$ method [4].

In Fig.(3(a)) is shown the measured momentum bias existing in the Inner Detector before the alignment. Biases can be seen in regions with $|\eta|>1$ while the barrel region of the detector shows no significant biases. Using this momentum bias map, we can correct the localized distortions performing a momentum constrained alignment. The result is shown in Fig. (3(b)). We can observe a significantly correction over the entire $\eta-\phi$ space.

\section{B. Measurement of detector distortions using $Z \rightarrow \mu \mu$}

By using a very clean sample of $Z \rightarrow \mu^{+} \mu^{-}$combined muons (muons reconstructed using the Inner Detector and the Muon Spectrometer), we can obtain the existing momentum bias in the Inner Detector and remove it in a similar manner we have done in the $E / p$ method.

As we have observed in the previous subsection, deformations manifest themselves as biases in the reconstructed track parameters, in particular the momentum, and it will in turn 
bias the reconstructed $Z$ mass throughout the different detector regions. This method is able to provide a momentum bias measurement fully complementary to the $E / p$ method.

The invariant mass $m$ of two low-mass and highly relativistic particles is given approximately by:

$$
m^{2}=2 p_{1} p_{2}(1-\cos \theta)
$$

where $p_{1}$ and $p_{2}$ are the momenta of the particles and $\theta$ is the angle between the momentum vectors. Ignoring the uncertaintis on the angular variables (which are negligible compared to the size of the momentum uncertainties at $Z$ mass scale), and using Eq. (6), the reconstructed mass $m_{r e c}$ can be expressed in terms of the momentum biases:

$$
\Delta\left(m^{2}\right) \equiv \frac{m_{r e c}^{2}-m_{Z}^{2}}{m_{Z}^{2}} \approx q_{1} p_{T 1} \delta_{\text {sagitta } 1}+q_{2} p_{T 2} \delta_{\text {sagitta } 2}
$$

In Fig.(4(a)) is shown the measured momentum bias before the alignment. We can observe that we have reproduced almost the same result we got with the $E / p$ method (biases in regions with $|\eta|>1$ while the barrel region of the detector shows no significant biases) which proves the complementarity of the two methods. Using this momentum bias map, we can correct the localized distortions performing a momentum constrained alignment. The corrected result is shown in Fig. (4(b)).

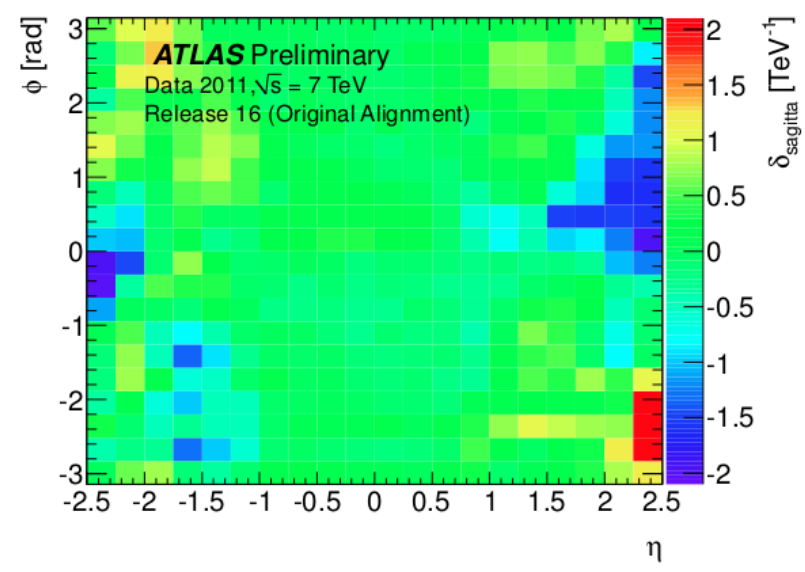

(a) Before alignment

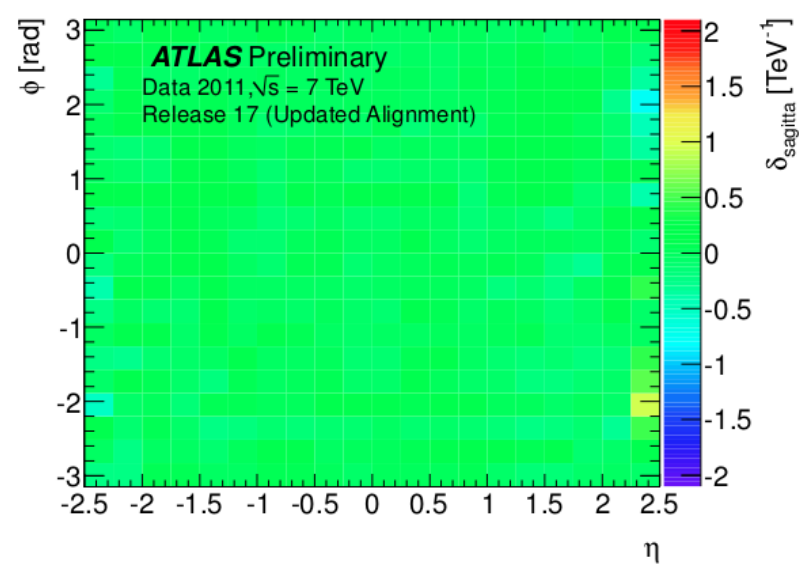

(b) After aligment

Fig. 4. Momentum bias measured before and after momentum constrained alignment. Using $Z \rightarrow \mu \mu$ method [4].

\section{Impact parameters bias}

Another observable we use to remove systematics and improve the alignment are the longitudinal $\left(z_{0}\right)$ and transversal $\left(d_{0}\right)$ impact parameter bias.

To obtain the impact parameters bias we also use the sample of $Z \rightarrow \mu^{+} \mu^{-}$combined muons we have use in the previous subsection.

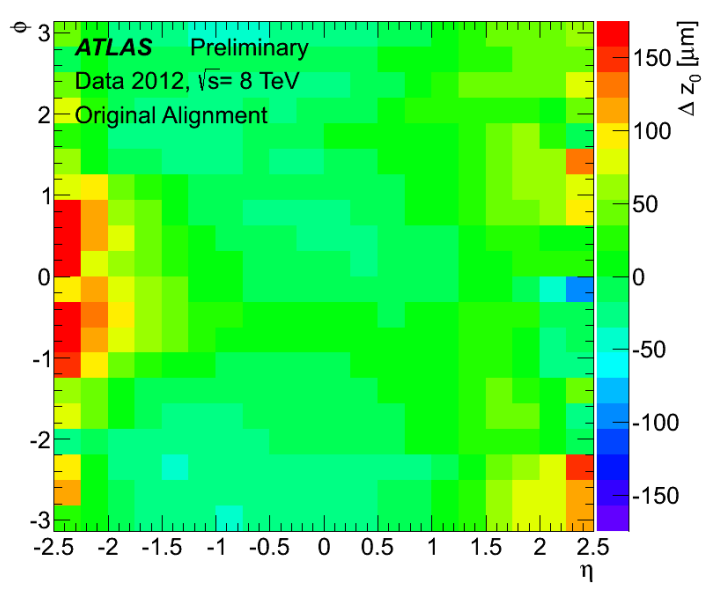

(a) Before alignment

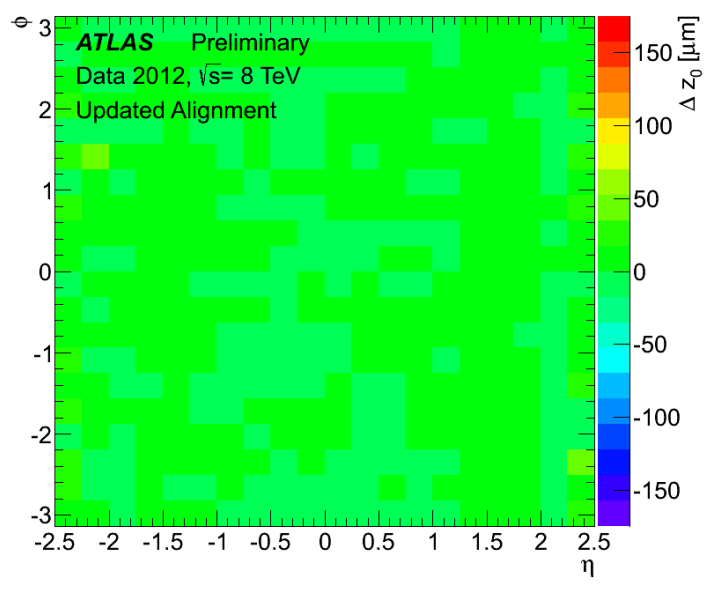

(b) After alignment

Fig. 5. $z_{0}$ bias as a function of $\eta$ and $\phi$ before and after the momentum and impact parameter constrain alignment [5].

The transversal and longitudinal impact parameter bias are measured by comparing the average distance between muons from a $\mathrm{Z}$ boson decay at the beam line.

$$
\begin{aligned}
& \delta_{d_{0}}=\left(d_{0}^{\mu_{1}}-d_{0}^{\mu_{2}}\right) \\
& \delta_{z_{0}}=\left(z_{0}^{\mu_{1}}-z_{0}^{\mu_{2}}\right)
\end{aligned}
$$

We can observe the $z_{0}$ and $d_{0}$ bias existing in the ID before the alignment (Fig. (5(a)), Fig. (6(a))). But using these bias maps as a constrain for the alignment procedure we can successfully minimize this distortion (Fig. (5(b)), Fig. (6(b))). 


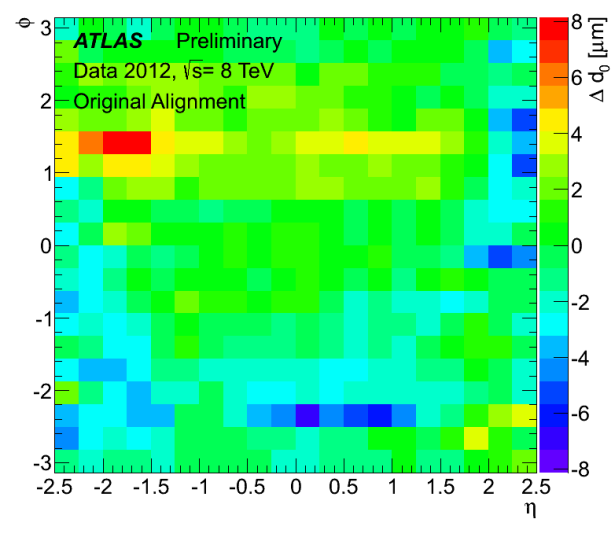

(a) Before alignment

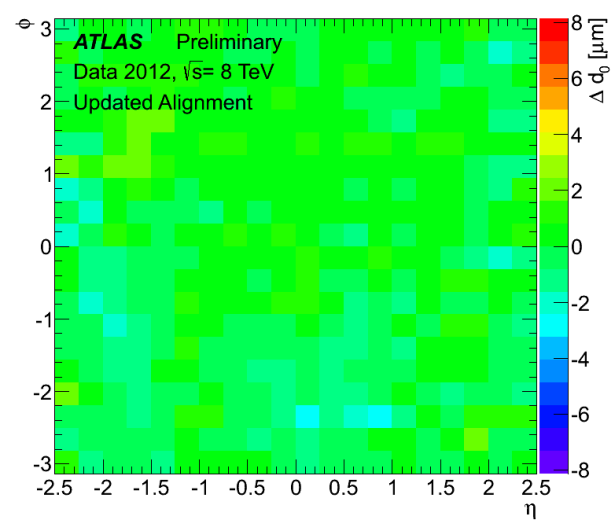

(b) After alignment

Fig. 6. $d_{0}$ bias as a function of $\eta$ and $\phi$ before and after the momentum and impact parameter constrain alignment [5].

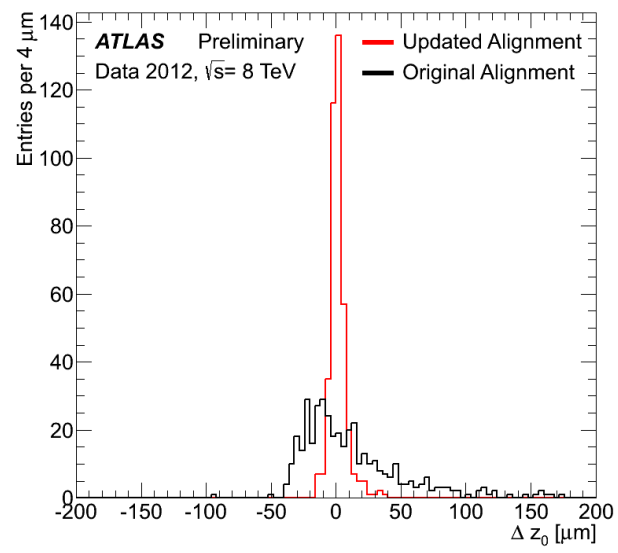

Fig. 7. Projection of the longitudinal impact parameter bias before and after the alignment [5].

Finally is shown the one-dimensional projection of the bias maps comparing the results between the original and updated alignment (Fig. (7), Fig. (8))

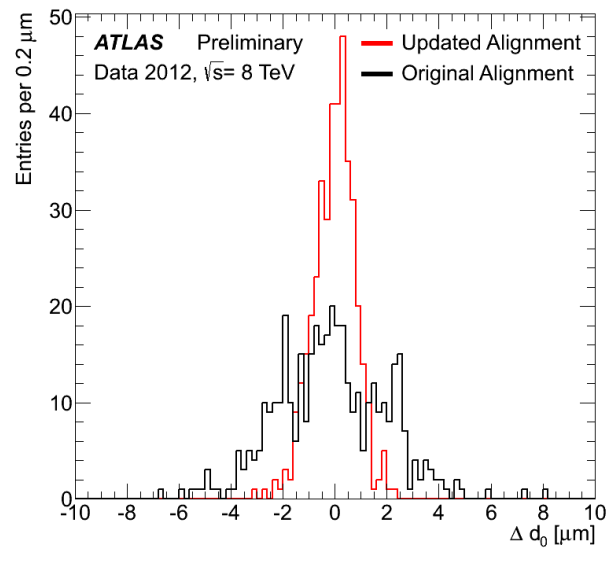

Fig. 8. Projection of the transversal impact parameter bias before and after the alignment [5].

\section{Conclusions}

The current status of the Inner Detector alignment has been presented. In order to achieve the ATLAS physics goals, the ID alignment has to reach a precise knowledge of modules positions. For this purpose we have developed the differents tools shown in this talk. The Level 1 run-by-run alignment helps to monitoring the detector behavior and prevent a degradation of the track parameteres for the data taking. Also we have shown that our tools (the $E / p$ method and the using of muons to extract the momentum and impact parameter bias), can measure localized distortions and successfully correct them. We have further improved the alignment of the ATLAS Inner Detecctor.

\section{REFERENCES}

[1] ATLAS Collaboration, "The ATLAS Experiment at the CERN Large Hadron Collider," J. Instrum., vol. 3, p. S08003. 437 p, 2008, Also published by CERN Geneva in 2010.

[2] Perez Cavalcanti, T, "Neural network based cluster creation in the ATLAS silicon Pixel Detector," CERN, Geneva, Tech. Rep. ATL-INDET-PROC2012-021, Nov 2012.

[3] ATLAS Collaboration, "Alignment of the ATLAS Inner Detector Tracking System with 2010 LHC proton-proton collisions at sqrts $=7 \mathrm{TeV}$," CERN, Geneva, Tech. Rep. ATLAS-CONF-2011-012, Mar 2011.

[4] ATLAS Collaboration, "Study of alignment related systematic effects on the ATLAS Inner Detector tracking," CERN, Geneva, Tech. Rep. ATLASCONF-2012-141, Oct 2012.

[5] A. K. Morley, P. Bruckman De Renstrom, and S. Pedraza Lopez, "Improved impact parameter biases," CERN, Geneva, Tech. Rep. ATLCOM-PHYS-2012-1520, Oct 2012. 